\title{
Mediadores inflamatorios: su relación con el dolor crónico y problemas asociados. Revisión bibliográfica
}

\author{
Inflammatory mediators: its connection with chronic pain \\ and associated problems. Review
}

L. Crespo-Pardo ${ }^{1}$ e Y. Taboada-Iglesias ${ }^{1,2}$

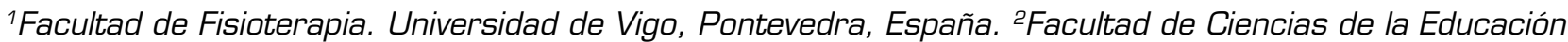
y del Deporte. Departamento de Didácticas Especiales. Universidad de Vigo, Pontevedra. Grupo de investigación en Educación, Actividad Física y Salud. (Gies10-DE3). Instituto de Investigación Sanitaria Galicia Sur (IIS Galicia Sur), SERGAS-UVIGO. Vigo, España

\section{RESUMEN}

Introducción: El dolor es una compleja interacción de sistemas, con un proceso que integra información sensorial, cognitiva y/o emocional proveniente de amenazas reales o potenciales. En el dolor crónico se ha observado una relación con otros síntomas somáticos y psicológicos como la depresión y la ansiedad, falta de sueño, fatiga y disminución de la capacidad cognitiva. Se ha demostrado que las citoquinas proinflamatorias y los síntomas asociados influyen en la probabilidad de padecer dolor crónico. El objetivo es analizar los niveles de los mediadores inflamatorios en patologías que cursan con dolor crónico y los trastornos relacionados para así comprender mejor el dolor de larga duración.

Material y métodos: Para la realización de este trabajo se realizó una revisión de la literatura científica mediante una búsqueda sistematizada en enero de 2020 en las bases de datos Pubmed, CINHAL, Medline, Scopus y Web of Sciencie. Se aplicaron como criterios de inclusión: ser documentos publicados en los últimos 5 años y estar escritos en inglés o castellano. Como criterios de exclusión: estudios de revisión, artículos repetidos en otras bases o que trataran sobre otro tema.

Resultados: 13 artículos fueron seleccionados tras aplicar los criterios de inclusión y exclusión. Todas las publicaciones son estudios observacionales. Se com-
Crespo-Pardo L, Taboada-Iglesias Y. Mediadores inflamatorios: su relación con el dolor crónico y problemas asociados. Revisión bibliográfica. Rev Soc Esp Dolor. 2021;28(1):37-46

\section{ABSTRACT}

Introduction: Pain is a complex interaction of systems, with a process that integrates sensory, cognitive and/or emotional information that comes from real or potential threats. It has been observed a connection in the chronic pain with other somatic and psychological symptoms, such as depression, anxiety, lack of sleep, fatigue and reduction of cognitive capacity. It has been demonstrated that proinflammatory cytokines and associated symptoms influence in the probability of suffering chronic pain. The objective is to analyze the levels of the inflammatory mediators in diseases with chronic pain and with related disorders, so that we can better understand long-term pain.

Material and methods: To make this dissertation, a review of the scientific literature was carried out by a systematized search in the Pubmed, CINHAL, Medline, Scopus and Web of Science databases, in January 2020. The inclusion criteria were: to be documents written in English or Spanish and published in the last 5 years. The exclusion criteria were: review studies, and articles repeated in other databases or that addressed other topics.

Results: 13 articles were selected after applying inclusion and exclusion criteria. All publications were observational studies. Proinflammatory cytokine values were compared in patients with chronic pain, taking
Recibido: 19-08-2020

Aceptado: 02-12-2020

Correspondencia: Yaiza Taboada-Iglesias yaitaboada@uvigo.es 
pararon los valores de citoquinas proinflamatorias en pacientes con dolor crónico, teniendo en cuenta el insomnio, la tolerancia al dolor, el catastrofismo, el género y el índice de masa corporal.

Conclusión: El dolor crónico está condicionado por múltiples factores, por lo que se debe conocer la fisiopatología del mismo. De esta manera, la medición de la proteína $\mathrm{C}$ reactiva y de citoquinas proinflamatorias podría suponer un avance en la evaluación y seguimiento del paciente con dolor crónico.

Palabras clave: Dolor crónico, citoquinas inflamatorias, insomnio, proteína $\mathrm{C}$ reactiva. into account insomnia, pain tolerance, catastrophism, gender and body mass index.

Conclusion: Chronic pain is conditioned by multiple factors, so its physiopathology must be known. In this way, the measurement of the C-reactive protein and the proinflammatory cytokines could mean an advance in the evaluation and monitoring of the patient with chronic pain.

Key words: Chronic pain, inflammatory cytokines, insomnia, C-reactive protein.

\section{INTRODUCCIÓN}

La causa de dolor musculoesquelético se ha relacionado a lo largo de la historia con un tejido lesionado que envía aferencias dolorosas hacia el sistema nervioso central para su percepción. Sin embargo, no siempre debe encontrarse relación entre el dolor y la nocicepción, ni entre el dolor y el daño de un tejido (1). Actualmente, la IASP (Asociación Internacional para el Estudio del Dolor) dice que "el dolor es una experiencia sensorial y emocional desagradable asociada con, o similar a la asociada con, daño tisular real o potencial" (ㄹ).

El dolor es definido como una compleja interacción de sistemas homeostáticos en respuesta a una amenaza identificada (프. De esta manera, se debe considerar como un proceso que integra información sensorial, cognitiva y/o emocional proveniente de amenazas reales o potenciales (4)]. Este proceso de evaluación de la amenaza es realizado por un conjunto de estructuras corticales y subcorticales denominadas neuromatriz del dolor (므. Sin embargo, debemos diferenciar el dolor agudo del dolor crónico (DC). La base del dolor agudo es la respuesta inflamatoria con la liberación de algógenos químicos ante un estímulo nocivo o daño tisular, como las prostaglandinas, bradicinina, el factor de necrosis tumoral (TNF), factor de crecimiento neural, histamina, sustancia $P$ y péptido relacionado con el gen de la calcitonina. Esta inflamación va a sensibilizar al nociceptor e incrementar la generación y transmisión de estímulos, lo que se conoce como sensibilización periférica, disminuyendo el umbral nociceptivo y facilitando las respuestas nociceptivas para promover la recuperación de los tejidos [트. En cambio, el DC se caracteriza por la sensibilización de la médula espinal, y la activación de las vías nociceptivas que se proyectan a la médula y mesencéfalo, así como la activación de los sistemas facilitadores descendentes del dolor y la pérdida de la inhibición descendente, para el mantenimiento de la sensibilización (ㄱ).

En líneas generales se ha demostrado que aquellas personas con problemas emocionales, de comportamiento, de consumo excesivo de alcohol o con trastor- nos de sueño, presentan mayores probabilidades de desarrollar dolor musculoesquelético a medio o largo plazo [으. De hecho, el DC tiene un efecto devastador en muchos aspectos de la vida diaria, disminuyendo la calidad de vida del paciente al repercutir negativamente en su salud física y emocional (9). Según una encuesta europea, la mitad de los pacientes con DC se sienten cansados todo el tiempo y el $40 \%$ indefensos o incapaces de pensar o funcionar con normalidad (10). Además, un 62 \% manifestaron sentir una falta de concienciación y conocimiento de la enfermedad en su entorno, y el $47 \%$ creían que el resto de la gente duda de la existencia real de su dolor (9).

Por otro lado, se ha definido una entidad clínica, el dolor crónico generalizado (DCG), que se define como dolor generalizado en el cuerpo, y que a menudo incluye otros síntomas somáticos y psicológicos como la depresión y la ansiedad, falta de sueño, fatiga y disminución de la capacidad cognitiva (11). Por ello, las creencias y emociones son capaces de activar la neuromatriz, provocar e incluso perpetuar el dolor sin la necesidad de la nocicepción (12).

Los problemas de sueño, incluyendo el insomnio, son una queja común entre los adultos con dolor crónico (13). Estas alteraciones se han asociado a niveles superiores de citoquinas inflamatorias circulantes (14), las cuales también varían como respuesta al estrés (15). Además, diversas investigaciones han confirmado la correlación positiva entre el catastrofismo, la intensidad del dolor y el grado de discapacidad percibida $(16,17)$.

Del mismo modo, el aumento de la sensibilidad al dolor experimental (hiperalgesia) se ha encontrado en condiciones de dolor crónico. En comparación con sujetos sanos, los pacientes con DC tienen bajos umbrales de dolor, lo que implica un cambio en las sensaciones percibidas con aumento del dolor ante estímulos no dolorosos (alodinia), disminución de la tolerancia al dolor y un aumento en los índices de dolor en respuesta a estímulos de dolor experimentales (18). En este sentido, la hiperalgesia generalizada se ha relacionado con la proteína $\mathrm{C}$ reactiva $\left(\mathrm{PC}_{\mathrm{R}}\right)$, revelando una asociación entre dicha proteína y la tolerancia al dolor 
experimental (19). Además, después de una lesión nerviosa periférica, los macrófagos y las células de Schwann, que se reúnen alrededor de la zona lesionada del nervio, secretan citocinas y factores de crecimiento específicos necesarios para la regeneración nerviosa. La irritación inflamatoria localizada del ganglio de la raíz dorsal (GRD) no solo aumenta las citocinas proinflamatorias, sino que también disminuye las citocinas antinflamatorias $(20,21)$.

La $P C_{R}$ se puede sintetizar en el hígado, y en otras células de tejidos como el riñón (르), pulmón (른 ), sis-

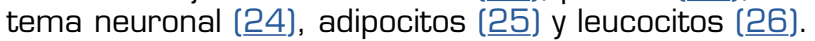
La $P_{\mathrm{R}}$ se expresa como resultado de varios estímulos, como por ejemplo IL-6 e IL-1, siendo considerada una proteína clásica de fase aguda. El nivel de $\mathrm{PC}_{\mathrm{R}}$ se correlaciona aceptablemente con la gravedad de la inflamación, lo que lo convierte en un marcador confiable para el seguimiento de las infecciones, inflamaciones y de la respuesta al tratamiento (르) y, por lo tanto, un marcador inflamatorio asociado al dolor.

En definitiva, pese a que el dolor agudo está tradicionalmente relacionado con la inflamación, el objetivo de este estudio es analizar el comportamiento de los mediadores inflamatorios (MI) en patologías que cursan con dolor crónico y los trastornos asociados a dicha enfermedad, de manera que podamos comprender el dolor de larga duración y todos los mecanismos que influyen en él, para así, abordarlo con mayor criterio.

\section{MATERIAL Y MÉTODOS}

Para la realización de este trabajo se realizó una búsqueda en Pubmed, Scopus, Web of Science, Medline y CINHAL en el mes de enero de 2020. Se utilizaron los mismos términos del Medical Subject Headings (MeSH) en las bases utilizadas: "Chronic Pain", "Inflammation", "Interleukin-6", "C-Reactive Protein" y "Chemokines" [Tabla I).

Para la obtención de los resultados válidos, se aplicaron como criterios de inclusión documentos publicados en los últimos 5 años y escritos en inglés o castellano. Como criterios de exclusión se estableció que fueran estudios de revisión, artículos repetidos en otra base de datos o trataran sobre diferente tema. Los resultados y el proceso de búsqueda se exponen en la Figura 1.

\section{RESULTADOS}

En la exposición de los resultados se ha realizado una tabla con el objetivo de facilitar la comprensión de las características metodológicas. Además, se ha pretendido establecer un orden lógico en función de los diferentes análisis y variables, de cara a facilitar su comprensión y posterior análisis. Dichas características metodológicas se establecen en la Tabla II.

Entre los principales resultados de los artículos incluidos, Sibille y cols. (20) señalaron que la $P C_{6}$ fue significativamente mayor entre los individuos con dolor crónico en comparación con aquellos sin dolor crónico $(p<0,001)$.

Del mismo modo, Feinberg y cols. (2의) destacaron que la media de $\mathrm{PC}_{\mathrm{B}}$ fue significativamente mayor en los casos de dolor crónico $(\dot{X}=5,54 \mathrm{mg} / \mathrm{I})$ en comparación con los controles $(\dot{X}=3,75 \mathrm{mg} / \mathrm{I})$. El nivel sérico de $P_{C_{R}}$ mostró una asociación positiva con el dolor crónico ( $p=0,0001)$.

Bäckryd y cols. (30) partieron de estudios donde se relaciona la sensibilización central en el dolor neuropático con la presencia de valores aumentados de citoquinas proinflamatorias. Observaron valores de IL-6 elevados en comparación con el grupo control. En cambio, no se observan diferencias significativas en IL-1 $\beta$ y IL-8.

En pacientes con dolor lumbar, Teodorezyk-Injeyan y cols. (31) encontraron que los niveles basales de IL-1 $\beta$, IL-6 y TNF, en comparación con controles asintomáticos, eran todos significativamente más altos en ambos grupos de pacientes con dolor lumbar $(p=0,0001$ a 0,003). En cambio, la producción de TNF aislada también era significativamente mayor en pacientes con dolor lumbar crónico frente a control y dolor agudo ( $p=0,003$ ). En pacientes con dolor lumbar crónico, los niveles de IL-1 $\beta$ y TNF se correlacionaron positivamente con las puntuaciones de dolor evaluadas ( $p<0,001)$. En ambos grupos de pacientes solo los niveles de IL-6 se correlacionaron significativamente con las puntuaciones de la escala de discapacidad ODI ( $p<0,001$ ).

TABLA I

ECUACIONES DE LAS BÚSQUEDAS BIBLIOGRÁFICAS

\begin{tabular}{|c|c|}
\hline Base de datos & Ecuación de búsqueda \\
\hline Pubmed & $\begin{array}{l}\text { ["Chronic Pain"[Mesh] AND "Inflammation"[Mesh]) AND ("Interleukin-6"[Mesh] OR "C-Reactive } \\
\text { Protein"[Mesh] OR "Chemokines"[Mesh]) }\end{array}$ \\
\hline CINHAL & $\begin{array}{l}\text { [“Chronic Pain” AND “Inflammation”] AND [“Interleukin-6” OR “C-Reactive Protein” OR } \\
\text { "Chemokines") }\end{array}$ \\
\hline Medline & $\begin{array}{l}\text { (MH "Chronic Pain") AND (MH "Inflammation") AND ((MH "Interleukin-6") OR (MH "C-Reactive } \\
\text { Protein") OR (MH "Chemokines")) }\end{array}$ \\
\hline Scopus & $\begin{array}{l}\text { KEY (“Chronic Pain" AND "Inflammation" AND (“Interleukin-6" OR "C-Reactive Protein" OR } \\
\text { "Chemokines")] }\end{array}$ \\
\hline Web of Science & $\begin{array}{l}\text { ("Chronic Pain" AND "Inflammation") AND ("Interleukin-6" OR "C-Reactive Protein" OR } \\
\text { "Chemokines") }\end{array}$ \\
\hline
\end{tabular}




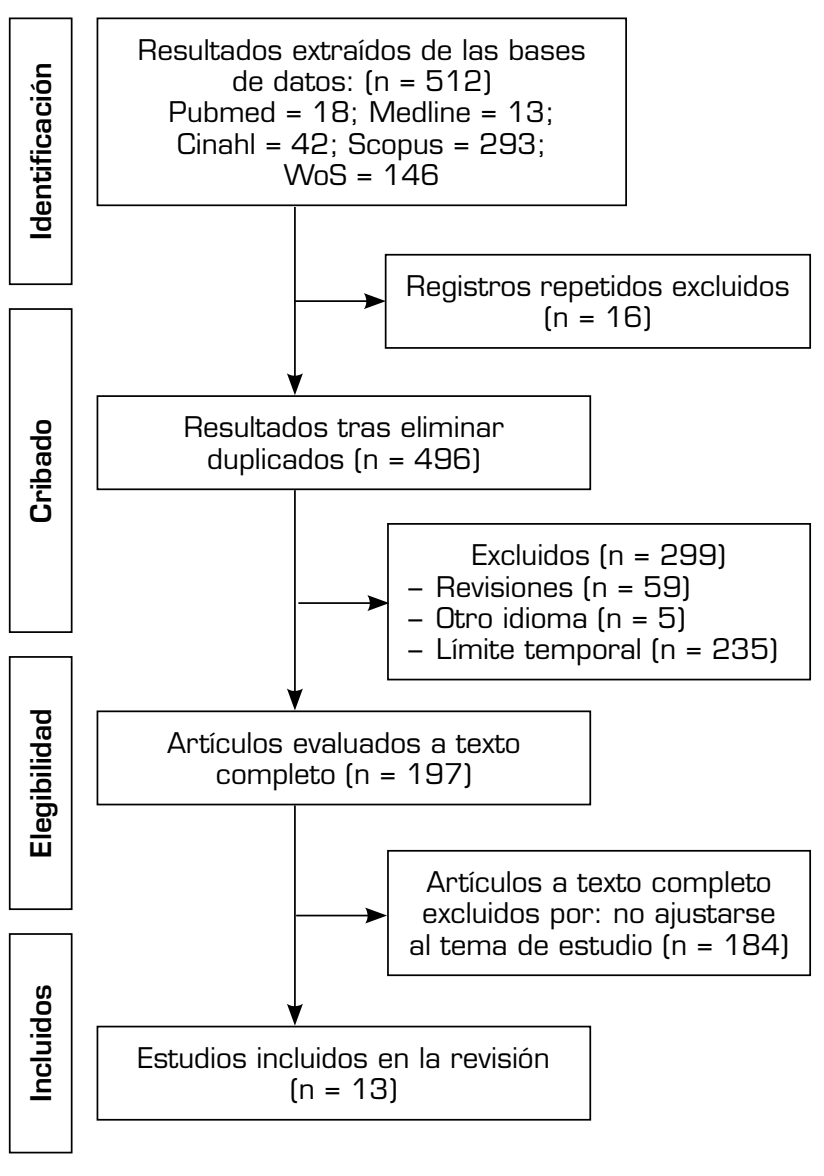

Fig. 1. Diagrama de selección.
Por su parte, Hysing y cols. (32]) analizaron 92 proteínas. Se obtuvieron valores de la $\mathrm{PC}_{\mathrm{P}}$ de 45 pacientes y presentaron un valor promedio de $2,6 \mathrm{mg} / \mathrm{I}$, lo que clasificaron como normal. Los valores basales de un total de 39 de marcadores inflamatorios del tipo dos cisteínas (CC) y quimiocinas CXC, que pertenecen al grupo de IL-8, fueron mayores en los 28 pacientes que participaron en la intervención durante un año de terapia conductual. Por otro lado, encontraron valores inferiores antes del tratamiento en las siguientes 4 citoquinas, el TNF, grupo de diferenciación 6 (CD6), IL-18 y oncostatina M (OSM).

Lasselin y cols. (33) aplicaron también terapia conductual, de la cual completaron 12 sesiones grupales con una duración de 90 minutos cada una, obteniendo una disminución significativa de las concentraciones séricas de TNF ( $p=0,02)$, pero no de IL-6 ni IL-8. En cambio, se observó que el tratamiento redujo la infelicidad psicológica (PIPS) y mejoró la calidad de vida relacionada con la salud mental (SF-36), pero solo en sujetos con un estado inflamatorio bajo de base respecto a los demás participantes.

Bäckryd y cols. (34) tuvieron como objetivo relacionar el riesgo de padecer neuropatía periférica y la asociación de valores aumentados de citoquinas. Analizaron 50 proteínas, de las cuales un $10 \%$ se asociaron significativamente con neuropatía periférica al compararlos con el grupo control. Entre ellas se encuentran la IL-8, las CC, las citoquinas relacionadas con factores de crecimiento y los factores inflamatorios.

En cuanto al estudio de Gerdle y cols. (11), se midieron los umbrales de dolor en sus extremidades superiores e inferiores con diferentes estímulos de presión, frío y calor, para registrar la intensidad del dolor y analizar su influencia sobre las proteínas inflamatorias. Han

TABLA II

CARACTERÍSTICAS DE LOS ARTÍCULOS ANALIZADOS

\begin{tabular}{|c|c|c|c|c|}
\hline Autor [es] & Tipo de estudio & Muestra & Criterios de selección & Herramienta/Variable \\
\hline $\begin{array}{l}\text { Sibille y cols. } \\
\text { [ㄹ] (2016] }\end{array}$ & $\begin{array}{l}\text { Epidemiológico } \\
\text { transversal }\end{array}$ & $\begin{array}{l}n=12.981 \text { [53,4 \% } \\
{[(+)]} \\
4 \text { grupos. De } 30 \text { a } 87 \\
\text { años }\end{array}$ & $\begin{array}{l}\text { Muestra aleatoria por } \\
\text { rangos de edades } \\
\text { (Troms } \varnothing]\end{array}$ & $\begin{array}{l}\text { HSCL [ansiedad y } \\
\text { depresión], } \\
\text { NRS [dolor] }\end{array}$ \\
\hline $\begin{array}{l}\text { Feinberg y } \\
\text { cols. [29] } \\
\text { [2017] }\end{array}$ & $\begin{array}{l}\text { Epidemiológico } \\
\text { transversal }\end{array}$ & $\begin{array}{l}\text { GE: } 1125 \text { (fibromialgia) } \\
\text { / GC: } 51.410 \\
52 \% \text { \% / } 69 \% \\
\text { sobrepeso }\end{array}$ & $\begin{array}{l}\mathrm{CE}:<18 \text { años, } \\
\text { embarazo, EA }\end{array}$ & $\begin{array}{l}\text { Inespecífica para el } \\
\text { insomnio }\end{array}$ \\
\hline $\begin{array}{l}\text { Bäckryd y } \\
\text { cols. [이] } \\
\text { [2016] }\end{array}$ & $\begin{array}{l}\text { Piloto. } \\
\text { Epidemiológico } \\
\text { transversal }\end{array}$ & GE: 14; GC: 19 & $\begin{array}{l}\text {-Cl: > } 18 \text { años, DC > } \\
6 \text { meses, < } 40 \text { mm en } \\
\text { VASPI } \\
\text {-CE: poca EDV, TTO, HTA }\end{array}$ & VASPI (dolor) \\
\hline $\begin{array}{l}\text { Teodorezyk- } \\
\text { Injeyan y } \\
\text { cols. (31)] } \\
\text { (2019] }\end{array}$ & $\begin{array}{l}\text { Epidemiológico } \\
\text { de casos y } \\
\text { controles }\end{array}$ & $\begin{array}{l}\text {-GE: } 22 \text { agudos, } 25 \text { DC } \\
\text {-GC: } 24\end{array}$ & $\begin{array}{l}\text {-Cl: } 22-60 \text { años, dolor } \\
\text { agudo > } 1 \text { mes, DC > } \\
3 \text { meses } \\
\text {-CE: < } 3 \text { en EVA, TTO } 15 \\
\text { días antes, EA }\end{array}$ & $\begin{array}{l}\text { EVA (dolor), ODI } \\
\text { (discapacidad) }\end{array}$ \\
\hline
\end{tabular}


TABLA II [CONT.]

CARACTERÍSTICAS DE LOS ARTÍCULOS ANALIZADOS

\begin{tabular}{|c|c|c|c|c|}
\hline Autor [es] & Tipo de estudio & Muestra & Criterios de selección & Herramienta/Variable \\
\hline $\begin{array}{l}\text { Hysing y } \\
\text { cols. (플] } \\
\text { [2019] }\end{array}$ & $\begin{array}{l}\text { Epidemiológico } \\
\text { transversal }\end{array}$ & $\begin{array}{l}\mathrm{n}=28, \text { el } 69 \% \text { con } \\
\text { depresión }\end{array}$ & $\begin{array}{l}\text {-Cl: fracaso en otras TC } \\
\text {-CE: alcohol, drogas, } \\
\text { trastornos psiquiátricos }\end{array}$ & $\begin{array}{l}\text { PEA (para analizar } \\
92 \text { proteínas } \\
\text { simultáneamente) }\end{array}$ \\
\hline $\begin{array}{l}\text { Lasselin y } \\
\text { cols. (33) } \\
\text { (2016) }\end{array}$ & $\begin{array}{l}\text { Piloto. Estudio } \\
\text { experimental }\end{array}$ & $n=41$ & $\begin{array}{l}\text {-Cl: dolor larga duración, } \\
\text { 18-65 años } \\
\text {-CE: recibir TTO, } \\
\text { episodios psiquiátricos }\end{array}$ & $\begin{array}{l}\text { EVA (dolor), PIPS (dolor), } \\
\text { SF-36 (calidad de vida), } \\
\text { HADS (ansiedad y } \\
\text { depresión) }\end{array}$ \\
\hline $\begin{array}{l}\text { Bäckryd y } \\
\text { cols. [34] } \\
\text { (2017) }\end{array}$ & $\begin{array}{l}\text { Epidemiológico } \\
\text { de casos y } \\
\text { controles }\end{array}$ & $\begin{array}{l}\text {-GE: } 511 \text { (X்: } 57 \text { años) } \\
\text {-GC: } 511 \text { [X்: } 54 \text { años] } \\
\text { +q en GC }\end{array}$ & $\begin{array}{l}\text {-Cl: > } 18 \text { años, } \\
\text { enfermedad crónica } \\
\text { ( }>6 \text { meses), > } 4 \text { en EVA }\end{array}$ & $\begin{array}{l}\text { PEA (para analizar } \\
92 \text { proteínas } \\
\text { simultáneamente) }\end{array}$ \\
\hline $\begin{array}{l}\text { Gerdle y } \\
\text { cols. (11) } \\
\text { [2017] }\end{array}$ & $\begin{array}{l}\text { Epidemiológico } \\
\text { transversal }\end{array}$ & $\begin{array}{l}\text {-GE: } 19 \text { con DC } \\
\text {-GC: } 24\end{array}$ & $\begin{array}{l}\text {-Cl: }+ \text {, } 20-65 \text { años. } \\
\text {-CE: fármacos, EA, } \\
\text { embarazo }\end{array}$ & $\begin{array}{l}\text { ACR (discapacidad), } \\
\text { NMCQ (dolor), HADS } \\
\text { (ansiedad y depresión), } \\
\text { PCS (catastrofismo), QOLS } \\
\text { (calidad de vida), PPT } \\
\text { (dolor) }\end{array}$ \\
\hline $\begin{array}{l}\text { Chamessian } \\
\text { y cols. [(포) } \\
\text { (2017) }\end{array}$ & $\begin{array}{l}\text { Epidemiológico } \\
\text { de casos y } \\
\text { controles }\end{array}$ & $\begin{array}{l}\text {-GE: } 36 \text { (amputados } \\
\text { con DC). GC: } 40 \\
\text { (amputados sin dolor) }\end{array}$ & $\begin{array}{l}\text {-Cl: + } 18 \text { años en } \\
\text { ejército, recibir TTO, } \\
\text { lesión } 3 \text { a } 18 \text { meses } \\
\text { antes de la inscripción. } \\
\text { CE: EA }\end{array}$ & $\begin{array}{l}\text { S-LANSS (dolor), } \\
\text { EVA (dolor), PCS } \\
\text { (catastrofismo) }\end{array}$ \\
\hline $\begin{array}{l}\text { Schistad y } \\
\text { cols. [36] } \\
\text { [2017] }\end{array}$ & $\begin{array}{l}\text { Epidemiológico } \\
\text { transversal }\end{array}$ & $\begin{array}{l}\mathrm{n}=10.274(51 \% \text { \%) } \\
4 \text { niveles PCR } \uparrow \mathrm{S}\end{array}$ & -CE: EA & HSCL (ansiedad y dolor) \\
\hline $\begin{array}{l}\text { Skarpsno y } \\
\text { cols. [37) } \\
\text { (2019) }\end{array}$ & $\begin{array}{l}\text { Cohorte } \\
\text { prospectivo }\end{array}$ & $\begin{array}{l}q=3214,0=3142< \\
1,0 \mathrm{mg} / \mathrm{l}, 1,00-2,99 \\
\mathrm{mg} / \mathrm{l}, \mathrm{y}>3,00 \mathrm{mg} / \mathrm{l}\end{array}$ & $\begin{array}{l}-\mathrm{CE}: \mathrm{DC}, \dot{\mathrm{X}}>10 \\
\mathrm{mg} \text { PCR } \uparrow \mathrm{S} \text {, poca } \\
\text { información sobre el } \\
\text { sueño }\end{array}$ & $\begin{array}{l}\text { Test inespecífico para } \\
\text { dolor e insomnio }\end{array}$ \\
\hline $\begin{array}{l}\text { Heffner y } \\
\text { cols. [ํㅛ] } \\
\text { [2018] }\end{array}$ & $\begin{array}{l}\text { Epidemiológico } \\
\text { de casos y } \\
\text { controles }\end{array}$ & $-G E=48(60 \%$ \% $)$ & $\begin{array}{l}\text {-Cl: } 50-75 \text { años, DC } \\
\text { (+ } 6 \text { meses) -CE: EA, } \\
\text { obesidad, menopausia }\end{array}$ & $\begin{array}{l}\text { CPT (tolerancia frío), PSG } \\
\text { (sueño), NFR (dolor), } \\
\text { ISI (insomnio), WOMAC } \\
\text { (osteoartritis), KPS (dolor } \\
\text { rodilla) }\end{array}$ \\
\hline $\begin{array}{l}\text { Park y cols. } \\
\text { (39) (2017) }\end{array}$ & $\begin{array}{l}\text { Epidemiológico } \\
\text { de casos y } \\
\text { controles }\end{array}$ & $\begin{array}{l}\text {-GE: } 20 \text { (dolor medio } \\
6 \text { sobre } 10 \text { ) }+20 \\
\text { (dolor medio } 2 \text { sobre } \\
\text { 10) } \\
\text {-GC: } 20 \text { ( } \text { (sin dolor) }\end{array}$ & $\begin{array}{l}\text {-CE: EA, fármacos } 4 \\
\text { meses antes }\end{array}$ & $\begin{array}{l}\text { GCPS (DC), PSQI (calidad } \\
\text { sueño), ESE (insomnio) }\end{array}$ \\
\hline
\end{tabular}

: mujer. n: muestra. HSCL: Hopkins Symptom Checklist. NRS: Numerical Rating Scale. GE: grupo exprimental. GC: grupo control. CE: criterios de exclusión. EA: enfermedades asociadas. Cl: criterios inclusión. DC: dolor crónico. VASPI: Visual Analog Scales of Pain Intensity. EDV: esperanza de vida. TTO: tratamiento. HTA: hipertensión arterial. EVA: escala visual analógica. ODI: Oswestry Disability Index. TC: terapia cognitiva. PEA: ensayo de extensión proximal multiplex. PIPS: escala de inflexibilidad psicológica ante el dolor. SF-36: calidad de vida relacionada con la salud mental. HADS: escala de ansiedad y depresión hospitalaria. ACR: Absolute Category Rating. NMCQ: cuestionario del ministerio del consejo nórdico. PCS: Pain Catastrophizing Scale. QOLS: escala de calidad de vida. PPT: presión por umbrales. S-LANSS: Leeds Assessment of Neuropathic Symptoms and Signs. PCR S: proteína C reactiva de alta sensibilidad. HSCL: Hopkins Symptom Checklist. ${ }^{\Uparrow}$ : hombre. Ẋ: media. CPT: prueba tolerancia al frío. PSG: polisomnografía. NFR: umbral nociceptivo de flexión refleja. ISI: índice de severidad del insomnio. WOMAC: índice de osteoartritis de Western Ontario. KPS: Knee Pain Scale. GCPS: Graded Chronic Pain Scale. PSQI: Pittsburgh Sleep Quality Index. ESE: escala de somnolencia de Epworth. 
encontrado diferencias significativas entre las intensidades de dolor entre grupos. Además, ambos grupos mostraron una mala calidad de vida, con una media alrededor de 38 puntos en la escala empleada. En cuanto a las citoquinas analizadas, 11 proteínas tuvieron diferencias significativas entre los grupos, entre las que se encuentra la IL-1 $\beta(p=0,026)$, distintos tipos de quimiocinas como la CCL2O ( $p=0,042)$, la CCL28 ( $p=0,051$ ) y la CCL4 ( $p=0,062)$, de las cuales solo la CCL2O obtuvo valores significativos.

Chamessian y cols. [35] mostraron niveles significativamente más altos de TNF, IL-8, PC ${ }_{R}$ y proteínas relacionadas con factores de crecimiento. Además, las puntuaciones promedio de gravedad de dolor (S-LANSS) y la escala catastrofización del dolor (PCS) se correlacionaron significativamente $(p<0,01)$ con el TNF, la IL-8, y las proteínas relacionadas con factores de crecimiento.

En siguiente lugar, el estudio de Schistad y cols. [36] tuvo como objetivo principal analizar la variabilidad de la proteína $C$ reactiva de alta sensibilidad $\left(\mathrm{PC}_{\mathrm{R \uparrow S}}\right)$ en pacientes con dolor crónico, y también cómo influía la tolerancia al frío en ella. Analizaron la ansiedad y el dolor con la escala de síntomas de Hopkins (HSCL), pero no encontraron diferencias significativas entre grupos. En relación a la $\mathrm{PC}_{\mathrm{R} \uparrow \mathrm{S}}$ los participantes con dolor crónico obtuvieron un nivel significativamente más alto (con valores de $P C_{\mathrm{R} s}>$ de $3 \mathrm{mg} / \mathrm{I}$ en el $18,5 \%$ de los participantes) en comparación con aquellos sin dolor $\left(\mathrm{PC}_{\mathrm{R} \uparrow \mathrm{S}}<\right.$ de $3 \mathrm{mg} / \mathrm{I}$ en el 1,7\%). Observaron una relación negativa entre los niveles de $\mathrm{PC}_{\mathrm{R} \uparrow \mathrm{S}}$ y la tolerancia al frío, ya que completaron el tiempo total de la prueba el $70,1 \%$ de las personas que se encontraban en el nivel leve de $P C_{\mathrm{R} s}(<1,0 \mathrm{mg} / \mathrm{l})$, el 69,1\% de los pacientes con nivel medio $(1,00-2,99 \mathrm{mg} / \mathrm{l})$, el 63,9\% de los que la tenían ligeramente elevada $[3,00-10 \mathrm{mg} / \mathrm{I}), \mathrm{y}$ solo el 61,2 \% de los que tenían niveles altos de $P C_{R \uparrow S}$ (> $10 \mathrm{mg} / \mathrm{l}$ ).

Por otro lado, Skarpsno y cols. (37) analizaron el riesgo de sufrir dolor crónico en función de los niveles de $\mathrm{PC}_{\mathrm{R} \uparrow \mathrm{S}}$ y de insomnio. El $18 \%$ de las personas con $\mathrm{PC}_{\mathrm{R} \uparrow \mathrm{S}}<1 \mathrm{mg} / \mathrm{I}$ tienen relación con elevado insomnio, y el $12 \%$ de sufrir dolor crónico. En niveles de 1,00$2,99 \mathrm{mg} / \mathrm{I}$ de $P \mathrm{C}_{\mathrm{prg}}$ presentaron en un $27 \%$ trastor-

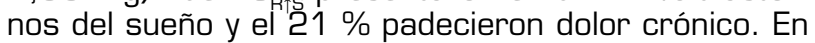
$P C_{\text {Prs }}>3,00 \mathrm{mg} / \mathrm{l}$ presentaron síntomas de los cuales el $31 \%$ estaba relacionado con falta de sueño y el $23 \%$ con el dolor crónico.

Heffner y cols. (38) basaron su objetivo en mejorar el insomnio con 6 semanas de terapia cognitivo-conductual (TCC-I) y evaluar su influencia posterior en los Ml en pacientes con osteoartristis de rodilla. Mostraron que los hombres ( $\dot{X}=16,33$ puntos) informaron de mayor gravedad del insomnio que las mujeres $(\dot{X}=24,27$ puntos], pero esta diferencia no resultó estadísticamente significativa $(p=0,04)$. A las 8 semanas de seguimiento, las puntuaciones de gravedad del insomnio en el grupo de TCC-I fueron significativamente más bajas que en el grupo control $(p=0,001)$. Compararon la mejora del insomnio con el Indice de osteoartritis de Western Ontario (WOMAC), Knee Pain Scale (KPS), la prueba de la tolerancia al frío (CPT), y la IL-6 y el TNF a través de la sesión de evaluación del dolor inicial. Las puntuaciones WOMAC disminuyeron en el grupo experimental 9,06 puntos, lo que sugiere un cambio importante en la clínica al mejorar el insomnio. La respuesta de la IL-6 en relación con el tiempo de mejora del insomnio fue significativa $(p=0,001)$. Los participantes que tenían mejoría del insomnio durante el seguimiento mostraron significativamente reducidos los niveles de la IL-6.

Park y cols. (39)] analizaron los $\mathrm{Ml}$ en pacientes con trastornos temporomandibulares (TTM) y cómo influye el insomnio en dichos valores. Los valores de la $P_{\mathrm{R}}$ fueron más altos en el grupo de mayor dolor, sin embargo, las diferencias entre grupos no fueron estadísticamente significativas ( $p>0,05)$. Pese a esto, los niveles de la IL-1 $\beta$, la IL-6 y el TNF sí difirieron significativamente entre los grupos ( $p<0,001$ para todos). El grupo de mayor dolor tenía los niveles más altos de todas las citoquinas proinflamatorias analizadas. Se encontraron diferencias significativas entre el grupo de mayor dolor y el grupo de control para la IL-6 ( $p<0,01)$ y entre los grupos con menor dolor y el grupo control para el TNF (p $<0,01\}$.

Las puntuaciones del sueño en la escala de somnolencia difirieron significativamente entre los grupos ( $p<0,001$ ), siendo las mayores puntuaciones en el grupo de mayor dolor.

\section{DISCUSIÓN}

Todos los artículos incluidos en esta revisión son estudios observacionales, debido a que se trata de un tema que se encuentra en las primeras etapas de investigación.

Gracias a las herramientas empleadas, niveles elevados de $\mathrm{Ml}$ se han correlacionado significativamente con niveles altos de discapacidad (31), con la infelicidad psicológica (33), con mala calidad de vida (33), con intensidades y gravedad de dolor altas $(11,35)$, con altos índices de catastrofización $(11,35)$ y con el insomnio) (37-39). Por lo que la asociación del dolor crónico con comorbilidades está presente y aumenta el riesgo de padecerlo.

\section{DOLOR Y MI}

Existe evidencia de que la IL-6 contribuye al desarrollo del comportamiento del dolor neuropático después de una lesión nerviosa periférica $(40,41)$. En el estudio de Bäckryd y cols. [ $\underline{30}$ ] se encuentran valores estadísticamente significativos de la IL-6 en pacientes con dolor neuropático. Sin embargo, un estudio de cohorte con mayor tamaño muestral indica que esta citoquina no muestra valores destacados un año después, donde encontraron aumentos en la CC y en la IL-8 (34), las cuales también han sido relacionadas con dolor neuropático [42].

A pesar de que la IL-6 sea la citoquina más estudiada en relación con el dolor nervioso, no es la que mayor valor significativo se muestra en el estudio de Chamessian y cols. [35], en donde los pacientes experimentales sufrían dolor crónico debido a una lesión por traumatismo del nervio en el muñón. Los niveles significativamente más altos fueron en el TNF, la IL-8, la $P C_{R}$ y las proteínas relacionadas con factores de crecimiento. Además, cabe destacar la importancia de que la $\mathrm{PC}_{\mathrm{R}}$ haya dado valores elevados, ya que, a pesar 
de ser considerada un marcador en fases tempranas de la inflamación, también se considera un marcador que permite la evaluar y monitorizar el desarrollo de la progresión inflamatoria $[27,43)$.

Existe abundante evidencia de que ciertas citocinas proinflamatorias como la IL-1 $\beta$, la IL-6 y el TNF están involucradas en el proceso del dolor patológico [ㄹ1]. Este grupo de mediadores han sido analizados en el estudio de Teodorezyk-Injeyan y cols. (31), en el cual al comparar pacientes con dolor lumbar crónico frente al grupo control, encontraron valores significativamente mayores en el grupo con dolor crónico. Además, el TNF aislado también obtiene diferencias significativas entre los pacientes con dolor lumbar crónico y agudo. Lasselin y cols. (33) también encuentran valores significativos, pero en este caso la terapia conductual redujo significativamente las concentraciones séricas de TNF. En ambos estudios se mostró una asociación entre el dolor y el TNF, ya que sus valores se reducen con la intervención o son menores en los pacientes que no tienen dolor. Cabe destacar que el TNF actúa en varias rutas de señalización a través de sus receptores presentes en las neuronas de la glía (44) y desempeña papeles importantes tanto en la hiperalgesia inflamatoria como en la neuropática (2己1).

Hysing y cols. [32] también apoyan el beneficio de la terapia conductual en las citoquinas del grupo al que pertenece la IL-8. Además, en este estudio aumentaron 4 citoquinas después del tratamiento. Este aumento, como el valor basal de quimiocinas que se expresan en fases agudas, puede ser debido a que el dolor crónico no es una inflamación en sí misma, como podrían considerarse las enfermedades reumatológicas como la osteoartritis [OA] o la espondilitis anquilosante. Además, en este estudio más de la mitad de los pacientes tienen problemas asociados como la depresión y/o la ansiedad, siendo procesos asociados que pueden contribuir a la liberación de citoquinas proinflamatorias en pacientes con dolor crónico, incluso en enfermedades no consideradas como inflamatorias.

Otro estudio en el que se realiza terapia conductual ha sido el de Heffner y cols. (38). En este el valor de la IL-6 se redujo de manera significativa tras la intervención. Uno de los criterios de inclusión era que los participantes debían tener evidencia médica de OA de rodilla y dolor durante más de 6 meses, por lo que en este caso se asocian las citoquinas inflamatorias con una enfermedad propiamente inflamatoria, pero de transcurso crónico.

Se ha observado en estudios con animales que la inflamación localizada en el GRD regula en exceso una serie de citocinas proinflamatorias, incluida la IL-6, e induce un brote simpático anormal en ausencia de lesión nerviosa periférica (20). En enfermedades inflamatorias como la OA el papel del sistema nervioso simpático puede aumentar la sensación dolorosa del paciente debido a la sensibilización periférica provocada por el estrés químico asociado al crecimiento nervioso perivascular (45).

Como ocurre en el dolor neuropático, los niveles de la IL-1 $\beta$, la IL-6 y el TNF también se ven aumentados en los pacientes con TTM del estudio de Park y cols. (39). Sin embargo, en estos, pese a tener valores más altos de $\mathrm{PC}_{\mathrm{R}}$ respecto al grupo control, los niveles de esta no son tan determinantes ni significativos. Los TTM se han correlacionado con hiperalgesia en zonas periféricas a la lesión (46), por lo que los valores de las citoquinas inflamatorias analizadas se entienden por el papel de estas en el proceso del dolor patológico [ㄹ1].

Por último, el dolor crónico se ha relacionado también con niveles aumentados y estadísticamente significativos de la $\mathrm{PC}_{\mathrm{B}}$ en los estudios de Sibille y cols. (28), Feinberg y cols. (르) y Skarpsno y cols. (3ㄱ). Dado el amplio número de participantes en los tres estudios y la relevancia de la $\mathrm{PC}_{\mathrm{R}}$ en los mecanismos del dolor, por estar influenciada por varias quimiocinas, se puede considerar como uno de los marcadores más relevantes y con más asociación al dolor crónico, ya que la $P C_{R}$ se ha relacionado con la hiperalgesia generalizada, revelando una asociación entre la tolerancia al dolor experimental y dicha proteína (19).

\section{TOLERANCIA AL DOLOR Y MI}

Las pruebas de tolerancia al dolor fueron empleadas por Gerdle y cols. (11), y los resultados guardaron relación con las citoquinas aumentadas, destacando entre ellas las quimiocinas de tipo CC y la IL-1 $\beta$, que regulan y activan la vía de señalización del dolor y, en concreto, la IL-1 $\beta$ aumenta la producción de sustancia $P$ en varias células neuronales y gliales (47), lo que va a aumentar la sensibilización periférica y por ello menor tolerancia al dolor (트). Esta asociación nos lleva a analizar el estudio de Schistad y cols. ( $\underline{36}$ ), donde los valores de $\mathrm{PC}_{\mathrm{R} \uparrow \widehat{S}}$ se correlacionaban con los pacientes de dolor crónico, además, toleraban menos el frío que el resto de los grupos. Teniendo en cuenta el gran tamaño de muestra de este estudio, con 10274 pacientes, y con los datos de Gerdle y cols. (11), se encuentra una asociación entre los Ml y pacientes de dolor crónico, así como con la disminución de la tolerancia al dolor.

\section{INSOMNIO Y MI}

El insomnio ha mostrado asociación con los Ml debido al aumento de estos cuando esa entidad clínica se acentúa $(13,14)$. Por ello, autores como Skarpsno y cols. (37), Heffner y cols. (38) y Park y cols. (39) analizaron la relación que podría haber en sus estudios.

Se observó que altos niveles de insomnio y valores de la $\mathrm{PC}_{\mathrm{R} T \mathrm{~S}}$ altos se vincula con mayor probabilidad de padecer dolor crónico (37). Park y cols. (39) también relacionó los peores indicadores de sueño con los grupos de mayor dolor y mayor puntuación de MI. No obstante, la terapia conductual se mostró como una técnica que mejora el insomnio y disminuye los valores de la IL-6 de manera significativa (38). La evidencia actual define la IL-6 como el mediador inflamatorio más sensible a los cambios de sueño (48).

Algunos estudios han demostrado que la restricción del sueño puede inducir una respuesta inflamatoria $(48,49)$, que puede contribuir a la sensibilización del sistema nociceptivo y de este modo aumentar la susceptibilidad para el desarrollo de dolor crónico (49).

En cuanto a la duración del sueño, el Ml que mayor asociación muestra es la $P C_{R}$. Se cree que la alteración del sueño tiene efectos proximales en la IL-6; pero como

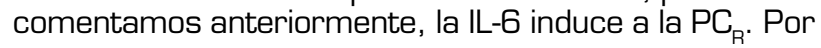


lo tanto, el aumento de la $\mathrm{PC}_{\mathrm{R}}$ podría deberse a una alteración del sueño más persistente o grave (48).

Es difícil sacar conclusiones sólidas de las alteraciones de sueño examinando tres artículos, dada la variedad de diferencias entre la metodología y marcadores inflamatorios. Pero a pesar de ello, la evidencia actual respalda los datos extraídos y, además, se ha descubierto que el tratamiento del insomnio reduce la inflamación $[50,51)$. Por lo tanto, el sueño debería ser una entidad clínica a considerar en el tratamiento de pacientes con dolor crónico.

\section{GÉNERO, INSOMNIO Y MI}

En ocho $(11,28,29,34,36,39)$ de los trece estudios de esta revisión, hay un mayor número de mujeres o solo estaba formado por ellas. El hecho de que haya un mayor número de mujeres podría ser porque los resultados señalan que las mujeres padecen mayor probabilidad de dolor crónico por factores como el ciclo menstrual, la sumación temporal del dolor, los mecanismos inhibitorios endógenos del dolor y las creencias y expectativas de género [르. Además, la historia individual pasada es influyente en la respuesta dolorosa en mujeres más que en hombres, en suma, otros factores psicosociales podrían contribuir a las diferencias en la sensibilidad del dolor entre hombres y mujeres. En el estudio de Sibille y cols. (28) el dolor crónico en mujeres fue significativamente más alto que en hombres, comparándolo con el grupo control.

Existen diferencias de sexo, con predominio femenino, en la calidad del sueño, la duración, la latencia y el insomnio (53). Sin embargo, las quejas de insomnio y somnolencia diurna son bastante más frecuentes en ellas, con un $58 \%$ frente a un $42 \%$ de los hombres (54). No obstante, Heffner y cols. (38) mostraron que sus pacientes masculinos padecían mayor insomnio respecto a las mujeres, a pesar de que esta diferencia no fue significativa. Pese a ello, parece que las mujeres pueden ser más vulnerables a los efectos del trastorno del sueño y muestran mayores aumentos de la IL-6 y la $\mathrm{PC}_{\mathrm{R}}$ (드).

\section{ANSIEDAD, DEPRESIÓN Y MI}

Una excitación del sistema inmune y el aumento de la secreción de los parámetros inmunes como la IL-6 y el TNF se asocian con un estado de ánimo negativo $(56,57)$. También se ha relacionado las mayores concentraciones de citoquinas y el hecho de padecer insomnio con el riesgo de padecer depresión (58-60).

Se ha propuesto que los factores que generan estrés ponen en marcha una cascada de sucesos bioquímicos que también implica la participación de Ml del sistema inmunológico, cooperando todos ellos en la aparición y expresión de la depresión (61).

De esta revisión se sugiere que la terapia conductual reduce las concentraciones de TNF, pero que en solo pacientes con estado inflamatorio bajo podría reducir la infelicidad (33). Asimismo, también produjo mejoras en los niveles inflamatorios en pacientes con ansiedad y depresión (32)]. A pesar de esto, la muestra en los dos estudios es pequeña, por lo que hay que ser prudentes en las afirmaciones.

Sibille y cols. (28) muestra que el dolor crónico no se correlacionó la depresión y la ansiedad. Schistad y cols. [ $\underline{36}$ ] tampoco encuentran relación con los niveles inflamatorios, utilizando la misma escala de valoración. Solamente se encuentra asociación entre la ansiedad, la depresión y los Ml en el estudio de Teodorezyk-Injeyan y cols. (31), por ello, a pesar de que la evidencia ha encontrado asociación entre los valores aumentados de $\mathrm{Ml}$ y el riesgo de sufrir dichos cuadros clínicos, los datos no son concluyentes.

Se concluye que existen valores estadísticos significativamente más altos en pacientes con dolor crónico frente al grupo control en alguna proteína o marcador inflamatorio analizado. A pesar de la variabilidad de los mediadores analizados, la $\mathrm{PC}_{\mathrm{R}}$ ha sido la proteína más analizada en todos los artículos, seguida de: el TNF, la IL-6, la IL-8 y la IL-1 $\beta$; ordenadas de mayor a menor número de aparición en los artículos. Un diagnóstico precoz ayudaría a mejorar el tratamiento.

De la misma manera se muestra asociación de padecer dolor crónico con valores aumentados de los MI, que a su vez disminuyen la tolerancia al dolor. También se relaciona con el insomnio, con mayor predominio en mujeres y obesidad. En contra de lo que dice la evidencia previa, no se ha mostrado asociación significativa con la ansiedad y la depresión en estos artículos.

\section{CONCLUSIONES}

Por lo tanto, la medición de la proteína C reactiva y de citoquinas proinflamatorias podría suponer un avance en la evaluación y el seguimiento del paciente con dolor crónico. Debido a la dificultad actual para realizar estas mediciones en la práctica clínica, el personal sanitario debe comprender el dolor y todos los mecanismos asociados que deberán ser considerados para un correcto abordaje y tratamiento del mismo.

\section{FINANCIACIÓN}

Este trabajo no se realizó mediante ninguna fuente de financiación, por lo que no existen conflictos de este tipo.

\section{BIBLIOGRAFÍA}

1. Wall PD, McMahon SB. Microneuronography and Its Relation to Perceived Sensation. A Critical Review. Pain. 1985;21(3):209-29. DOI: 10.1016/0304-3959(85) 90086-7.

2. Raja S, Carr D, Cohen M, Finnerup N, Flor H, Gibson S, et al. The revised International Association for the Study of Pain definition of pain: concepts, challenges, and compromises. Pain. 2020;161(9):1976-82. DOI: 10.1097/j. pain.0000000000001939.

3. Backonja MM. Defining Neuropathic Pain. Anesth Analg. 2003;97(3):785-90. DOl: 10.1213/01.ane.0000062826. 70846.8d. 
4. National Institute for Health and Clinical Excellence. Neuropathic Pain: The Pharmacological Management of Neuropathic Pain in Adults in Non-Specialist Settings. Clinical Guildeline 96. London: NICE; 2010.

5. Moseley GL. A Pain Neuromatrix Approach to Patients with Chronic Pain. Man Ther. 2003;8(3):130-40. DOI: 10.1016/ s1356-689x(03)00051-1.

6. García-Andreu J. Manejo Básico Del Dolor Agudo y Crónico. Anest Mex. 2017;29(1).

7. Zegarra JW. Bases Fisiopatológicas Del Dolor. Acta Med Per. 2007;24(2):35-8.

8. Jussila L, Paananen M, Näyhä S, Taimela S, Tammelin T, Auvinen J, et al. Psychosocial and Lifestyle Correlates of Musculoskeletal Pain Patterns in Adolescence: A 2-Year Follow-up Study. Eur J Pain. 2014;18(1):139-46. DOI: 10.1002/j.1532-2149.2013.00353.x.

9. Langley PC, Ruiz-lban MA, Molina JT, De Andres J, Castellón JR, et al. The Prevalence, Correlates and Treatment of Pain in Spain. J Med Econ. 2011;14(3):367-80. DOI: 10.3111/13696998.2011.583303.

10. Breivik H, Collett B, Ventafridda V, Cohen R, Gallacher D. Survey of Chronic Pain in Europe: Prevalence, Impact on Daily Life, and Treatment. Eur J Pain. 2006;10(4):287-333. DOl: 10.1016/j.ejpain.2005.06.009.

11. Gerdle B, Ghafouri B, Ghafouri N, Bäckryd E, Gordh T. Signs of Ongoing Inflammation in Female Patients with Chronic Widespread Pain: A Multivariate, Explorative, Cross-Sectional Study of Blood Samples. Medicine. 2017;96(9): e6130. DOI: 10.1097/MD.0000000000006130.

12. Brosschot JF. Cognitive-Emotional Sensitization and Somatic Health Complaints. Scand J Psychol. 2002;43(2):113-21. DOI: 10.1111/1467-9450.00276.

13. Allen KD, Renner JB, Devellis B, Helmick CG, Jordan JM. Osteoarthritis and Sleep: The Johnston Country Osteoarthritis Project. J Rheumatol. 2008;35(6):1102-107.

14. Burgos I, Richter L, Klein T, Fiebich B, Feige B, Lieb K, et al. Increased Nocturnal Interleukin-6 Excretion in Patients with Primary Insomnia: A Pilot Study. Brain Behav. Immun. 2006;20(3):246-53. DOI: 10.1016/j.bbi.2005.06.007.

15. Prather AA, Puterman E, Epel ES, Dhabhar FS. Poor Sleep Quality Potentiates Stress-Induced Cytokine Reactivity in Postmenopausal Women with High Visceral Abdominal Adiposity. Brain. Behav. Immun. 2014;35:155-62. DOI: 10.1016/j.bbi.2013.09.010.

16. Blyth FM, March LM, Brnabic AJ, Cousins MJ. Chronic Pain and Frequent Use of Health Care. Pain. 2004;111(1-2):518. DOI: 10.1016/j.pain.2004.05.020.

17. Chatkoff DK, Leonard MT, Maier KJ. Pain Catastrophizing Differs Between and Within West Haven-Yale Multidimensonal Pain Inventory (MPI) Pain Adjustment Classifications. Clin J Pain. 2015;31(4):349-54. DOI: 10.1097/ AJP.0000000000000117.

18. Kaunisto MA, Jokela R, Tallgren M, Kambur O, Tikkanen E, Tasmuth T, et al. Pain in 1,000 Women Treated for Breast Cancer: A Prospective Study of Pain Sensitivity and Postoperative Pain. Anesthesiology. 2013;119(6):1410-21. DOl: 10.1097/ALN.0000000000000012.

19. Afari N, Mostoufi S, Noonan C, Poeschla B, Succop A, Chopko L, et al. C-Reactive Protein and Pain Sensitivity: Findings from Female Twins. Ann Behav Med. 2011;42(2):277-83. DOI: 10.1007/s12160-011-9297-6.

20. Xie WR, Deng H, Li H, Bowen TL, Strong JA, Zhang JM. Robust increase of cutaneous sensitivity, cytokine production and sympathetic sprouting in rats with localized inflammatory irritation of the spinal ganglia. Neuroscien- ce. 2006;142(3):809-22. DOI: 10.1016/j.neuroscience.2006.06.045.

21. Zhang JM, Jianxiong $A$. Cytokines, Inflammation and Pain. Int Anesthesiol Clin. 2007;45(2):27-37. DOI: 10.1097/ AlA.Ob013e318034194e.

22. Jabs WJ, Lögering BA, Gerke P, Kreft B, Wolber EM, Flinger MHF, et al. The Kidney as a Second Site of Human C-Reactive Protein Formation in Vivo. Eur J Immunol. 2003;33(1):15261. DOI: 10.1002/immu.200390018.

23. Ramage L, Proudfoot L, Guy K. Expression of C-Reative Protein in Human Lung Epithelial Cells and Upregulation by Cytokines and Carbon Particles. Inhal Toxicol. 2004;16(9):607-13.

24. Yasojima K, Schwab C, McGeer EG, McGeer PL. Human Neurons Generate C-Reactive Protein and Amyloid P: Upregulation in Alzheimer's Disease. Brain Res. 2000;887(1):80-9. DOI: 10.1016/s0006-8993(00)02970-x.

25. Calabro P, Chang DW, Willerson JT, Yeh ET. Release of C-Reactive Protein in Response to Inflammatory Cytokines by Human Adipocytes: Linking Obesity to Vascular Inflammation. J Am Coll Cardiol. 2005;46(6):1112-3. DOI: 10.1016/j. jacc.2005.06.017.

26. Kuta AE, Baum LL. C-Reactive Protein Is Produced by a Small Number of Normal Human Peripheral Blood Lymphocytes. J Exp Med. 1986;164(1):321-6. DOl: 10.1084/jem.164.1.321.

27. Du Clos TW, Mold C. C-Reactive Protein: An Activator of Innate Immunity and a Modulator of Adaptive Immunity. Immun Res. 2004;30(3):261-77. DOI: 10.1385/IR:30:3:261.

28. Sibille KT, Steingrímsdóttir OA, Fillingim RB, Stubhaug A, Schirmer $\mathrm{H}$, Chen $\mathrm{H}$, et al. Investigating the Burden of Chronic Pain: An Inflammatory and Metabolic Composite. Pain Res. 2016;2016:7657329. DOl: 10.1155/2016/7657329.

29. Feinberg T, Sambamoorthi U, Lilly C, Innes KK. Potential Mediators between Fibromyalgia and C-Reactive Protein: Results from a Large U.S. Community Survey. BMC Musculoskelet. Disord. 2017;18(1):294. DOl: 10.1186/s12891017-1641-y.

30. Bäckryd E, Ghafouri B, Larsson B, Gerdle B. Plasma ProInflammatory Markers in Chronic Neuropathic Pain: A Multivariate, Comparative, Cross-Sectional Pilot Study. Scand J Pain. 2016;10(1):1-5. DOI: 10.1016/j.sjpain.2015.06.006.

31. Teodorczyk-Injeyan JA, Triano JJ, Injeyan HS. Nonspecific Low Back Pain: Inflammatory Profiles of Patients With Acute and Chronic Pain Clin J Pain. 2019;35(10):818-25. DOI: 10.1097/AJP.0000000000000745.

32. Hysing EB, Smith L, Thulin M, Karlsten R, Bothelius K, Gordh T. Detection of Systemic Inflammation in Severely Impaired Chronic Pain Patients and Effects of a Multimodal Pain Rehabilitation Program. Scand J Pain. 2019;19(2):235-44. DOI: 10.1515/sjpain-2018-0340.

33. Lasselin J, Kemani MK, Kanstrup M, Olsson GL, Axelsson J, Andreasson A, et al. Low-Grade Inflammation May Moderate the Effect of Behavioral Treatment for Chronic Pain in Adults. J Behav Med. 2016;39(5):916-24. DOI: 10.1007/ s10865-016-9769-z.

34. Bäckryd E, Lind AL, Thulin M, Larsson A, Gerdle B, Gordh T. High Levels of Cerebrospinal Fluid Chemokines Point to the Presence of Neuroinflammation in Peripheral Neuropathic Pain: A Cross-Sectional Study of 2 Cohorts of Patients Compared with Healthy Controls. Pain. 2017;158(12):2487-95. DOI: 10.1097/j.pain.0000000000001061.

35. Chamessian A, Van de Ven T, Buchheit T, Hsia HL, McDuffie M, Gamazon ER, et al. Differential Expression of Systemic Inflammatory Mediators in Amputees with Chronic Residual Limb Pain. Pain. 2017;158(1):68-74. DOl: 10.1097/j. pain.0000000000000728. 
36. Schistad El, Stubhaug A, Furberg AS, Engdahl BL, Nielsen CS. C-Reactive Protein and Cold-Pressor Tolerance in the General Population: The Tromsø Study. Pain. 2017;158(7):1280-8. DOI: 10.1097/j.pain.0000000000000912.

37. Skarpsno ES, Mork PJ, Nilsen TI, Steingrímsdóttir ÓA, Zwart JA, Nilsen KB. The Interplay between Sleeplessness and High-Sensitivity C-Reactive Protein on Risk of Chronic Musculoskeletal Pain: Longitudinal Data from the Tromsø Study. Sleep. 2019;42(9):127. DOI: 10.1093/sleep/zsz127.

38. Heffner KL, France CR, Ashrafioun L, Quiñones M, Walsh P, Maloney MD, et al. Clinical Pain-Related Outcomes and Inflammatory Cytokine Response to Pain Following Insomnia Improvement in Adults With Knee Osteoarthritis. Clin J Pain. 2018;34(12):1133-40. DOI: 10.1097/ AJP.0000000000000644.

39. Park J, Chung J. Inflammatory Cytokines and Sleep Disturbance in Patients with Temporomandibular Disorders. Oral Facial Pain Headache. 2016;30(1):27-33. DOI: 10.11607/ ofph. 1367.

40. De Leo JA, Colburn RW. The Role of Cytokines in Nociception and Chronic Pain. In: Weinstein JN, Gordon SL, Editors. Low Back Pain: A Scientific and Clinical Overview. Illinois: American Academy of Orthopaedic Surgeons; 1995. p. 163-85.

41. Ramer MS, Murphy PG, Richardson PM, Bisby MA. Spinal Nerve Lesion-Induced Mechanoallodynia and Adrenergic Sprouting in Sensory Ganglia Are Attenuated in Interleukin-6 Knockout Mice. Pain. 1998;78(2):115-21. DOI: 10.1016/ s0304-3959(98)00121-3.

42. Old EA, Clark AK, Malcangio M. The Role of Glia in the Spinal Cord in Neuropathic and Inflammatory Pain. Handb Exp Pharmacol. 2015;227:145-70. DOl: 10.1007/978-3-66246450-2 8.

43. Yao ZY, Zhang Y, Wu H. Regulation of C-reactive protein conformation in inflammation. Inflammation Research. 2019;68(10):815-23. DOI: 10.1007/s00011-01901269-1.

44. Boka G, Anglade P, Wallach D, Javoy-Agid F, Agid Y, Hirsch EC. Immunocytochemical Analysis of Tumor Necrosis Factor and Its Receptors in Parkinson's Disease. Neuroscience Letters. 1994;172(1-2):151-4. DOI: 10.1016/03043940(94)90684-x.

45. Grässel S, Muschter D. Peripheral Nerve Fibers and Their Neurotransmitters in Osteoarthritis Pathology. Int J Mol Sci. 2017;18(5):931. DOI: 10.3390/ijms18050931.

46. Smith MT, Wickwire EM, Grace EG, Edwards RR, Buenaver LF, Peterson S, et al. Sleep Disorders and Their Association with Laboratory Pain Sensitivity in Temporomandibular Join Disorder. Sleep. 2009;32(6):779-90. DOI: 10.1093/ sleep/32.6.779.

47. Jeanjean AP, Moussaoui SM, Maloteaux JM, Laduron PM. Interleukin-1 Beta Induces Long-Term Increase of AxonaIly Transported Opiate Receptors and Substance P. Neuroscience. 1995;68(1):151-7. DOI: 10.1016/03064522(95)00106-s.

48. Irwin MR, Olmstead R, Judith E, CarrollJE. Sleep Disturbance, Sleep Duration, and Inflammation: A Systematic Review an Meta-Analysis of Cohort Studies and Experimental Sleep Deprivation. Biol Psychiatry. 2016;80(1):40-52. DOI: 10.1016/j.biopsych.2015.05.014.
49. Haack M, Sanchez E, Mullington JM. Elevated Inflammatory Markers in Response to Prolonged Sleep Restriction Are Associated with Increased Pain Experience in Healthy Volunteers. Sleep. 2007;30(9):1145-52. DOI: 10.1093/ sleep/30.9.1145.

50. Irwin MR, Olmstead R, Carrillo C, Sadeghi N, Breen EC, Witarama T, et al. Cognitive Behavioral Therapy vs. Tai Chi for Late Life Insomnia and Inflammatory Risk: A Randomized Controlled Comparative Efficacy Trial. Sleep. 2014;37(9):1543-52. DOI: 10.5665/sleep.4008.

51. Irwin MR, Olmstead R, Breen EC, Witarama T, Carrillo C, Sadeghi N, et al. Cognitive Behavioral Therapy and Tai Chi Reverse Cellular and Genomic Markers of Inflammation in Late Life Insomnia: A Randomized Controlled Trial. Biol Psychiatry. 2015;78(10):721-9. DOI: 10.1016/j.biopsych.2015.01.010.

52. Racine M, Tousignant-Laflamme Y, Kloda LA, Dion D, Dupuis G, Choinire M. A Systematic Literature Review of 10 Years of Research on Sex/Gender and Pain Perception - Part 2: Do Biopsychosocial Factors Alter Pain Sensitivity Differently in Women and Men? Pain. 2012;153(3):619-35. DOI: 10.1016/j.pain.2011.11.026.

53. Monica P, Christine L. Exploring Sex and Gender Differences in Sleep Health: A Society for Women's Health Research Report. J Womens Health. 2014;23(7):553-62. DOI: 10.1089/jwh.2014.4816.

54. Regal AR, Amigo MC, Cebrian E. El Sueño y Mujer. Rev Neurology. 2009;49:376-82. DOI: 10.33588/rn.4907. 2009041

55. Prather AA, Epel ES, Cohen BE, Neylan TC, Whooley MA. Gender Differences in the Prospective Associations of Self-Reported Sleep Quality with Biomarkers of Systemic Inflammation and Coagulation: Findings from the Heart and Soul Study. J Psychiatr Res. 2013;47(9):1228-35. DOI: 10.1016/j.jpsychires.2013.05.004.

56. Dinan TG. Inflammatory Markers in Depression. Curr Opin Psychiatry. 2009;22(1):32-6. DOI: 10.1097/YCO. Ob013e328315a561.

57. Rief W, Pilger F, Ihle D, Bosmans E, Egyed B, Maes M. Immunological Differences between Patients with Major Depression and Somatization Syndrome. Psychiatry Res. 2001;105(3):165-74. DOI: 10.1016/s0165-1781(01) 00338-9.

58. Dowlati Y, Herrmann N, Swardfager W, Liu H, Sham L, Reim EK, et al. A Meta-Analysis of Cytokines in Major Depression. Biol Psychiatry. 2010;67(5):446-57. DOI: 10.1016/j.biopsych.2009.09.033.

59. Howren MB, Lamkin DM, Suls J. Associations of Depression with C-Reactive Protein, IL-1, and IL-6: A Meta-Analysis. Psychosom Med. 2009;71(2):171-86. DOI: 10.1097/ PSY.Ob013e3181907c1b.

60. Almeida OP, Alfonso H, Yeap BB, Hankey G, Flicker L. Complaints of Difficulty to Fall Asleep Increase the Risk of Depression in Later Life: The Health in Men Study. J Affect Disord. 2011;134(1-3):208-16. DOI: 10.1016/j.jad.2011. 05.045.

61. Anisman $\mathrm{H}$. Cascading Effects of Stressors and Inflammatory Immune System Activation: Implications for Major Depressive Disorder. J Psychiatry Neurosci. 2009;34(1):4-20. 